Schweiz. Z. Path. Bakt. 1956;19:802-812

\title{
Register rerum ad Vol. XIX
}

(B) $=$ Buchbesprechung - Livre nouveau - Book review

$(\mathrm{V})=$ Vortrag - Communication - Report

Angaben in Klammern () = Die Arbeit ist nicht erschienen oder wird an eine $\Gamma$

anderen Stelle publiziert werden. Indications entre parentheses ()$=$ Le travail n'a pas paru ou sera publié ailleurs. Notes in parentheses ()$=$ The paper has not been published or will appear elsewhere.

Bearbeitet von G. Boehm, Basel.

Agglutination, v. Antigène T,

Listeriose Agglutinine, v. Bang'-Agglutinine Allergie; gleichzeitiges Einsetzen von

Allergie und Tuberkelbildung bei

d. Meerschweinchentuberkulose, 1 -; nekrotisierende Arteriitis nach

Impfungen und ihre Deutung im

Rahmen der heutigen Allergie-

lehre, 411 Allergy; migraine and vascular

allergy, 692 (B) Alzheimer; morbus Alzheimer and

morbus Pick, a genetic, clinical a.

patho-anatomical study, 244 (B) Amino acids, v. Tetanus toxin Amnionflüssigkeit; Einfluß einer sta-

bilisierten Amnionflüssigkeit auf

d. regenerierende Rattenleber nach

partieller Hepatektomie, 288 Amnionhaltige Salbe, Beschleuni-

gung der Wundheilung beim Meer-

schweinchen, 729 Amnion-Salbe, stabilisierte, Stimulation der senilen Rattenepidermis,

429 Anatomie, v. Pathologische Anato-

mie Anatomopathologistes, v. Congrès Anthraxfraktionen, v. Bacillus

anthracis Antibacterial antibodies, natural, specificity, 189 Antibakterielle Substanzen, v. Coli-

cines Antibiothérapie, v. Antibiotiques Antibiotica et Chemotherapeutica,

Fortschritte (Vol. 2), 366 (B) Antibiotica, v. Pikromycin-

Resistenz, Streptomycin

Antibiotiques; les nouveaux antibiotiques et Torientation actuelle de Tantibiothérapie, 798 (B)

Antibodies, antibacterial, natural, specificity, 189

-, v. Plague

Anticorps, v. Poliomyelitis

Antigen-Antikörper Reaktion, v. Arteriitis

Antigène, v. Colistämme, Prozonen-mechanismus

Antigène «O

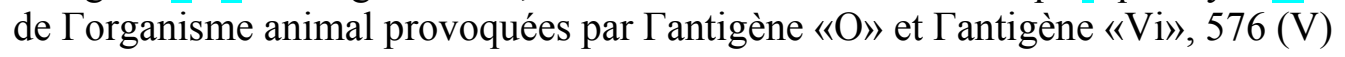


Antigène $M$ et $C$; action de certains anions tensio-actifs sur le com-portement antigénique des consti-tuants $\mathrm{M}$ et $\mathrm{C}$ des streptocoques hémolytiques du groupe $\mathrm{A}$, Ill

Antigène $\mathrm{T}$; influence de certains anions tensio-actifs sur les reactions d'agglutination spécifique étudiées sur le système $\mathrm{T}$ des streptocoques hémolytiques de groupe $\mathrm{A}, 519$ (V)

Antigène «Vi», v. Antigène «O»

Anti-ínositols; recherches sur les anti-inositols. Action de Гisomyti-litol sur

Schizosaccharomyces Pombe (Lindner), souche liquefa-ciens (Osterwalder) Dekker, 647 (V)

-, v. Cyclitols

Antikörper, inkomplette, in der Im-munbiologie, 247 (B)

-, v. Prozonenmechanismus

Antiphagenwirkung; Versuch zur

Register rerum ad Vol. XIX

803

Analyse der direkten und indi-

Tekten Antiphagenwirkung, 331 Arteriitis, nekrotisierende, nach Imp-

fungen und ihre Deutung im Rah-

men der heutigen Allergielehre, 411 Arthritis, v. Gelenkveränderungen,

Hypovitaminose C, Streptokokken Articulationes; lesions articulaires et

périarticulaires de Thypovitami-

nose $\mathrm{C}$ chronique chez le cobaye,

369 Ascorbinsäure, v. Hypovitaminose C Aspirationsbiopsie, v. Organpunktion Autonomes

Nervensystera, v. Patho-

logische Anatomie Azofarbstoffe, v. Cancerogene Wir-

kung

Bacille tuberculeux, recherche, isole-ment et etude, 801 (B)

Bacillus anthracis; Wirkung von Anthraxfraktionen auf mesenchy-male Zellreaktionen in vitro und in vivo, $639(\mathrm{~V})$

Bacillus anthracis (cereus, megate-ríum), v. Zellwand gewisser Ba-zillen

Bactericidal action, v. Gram-stain

Bacteries lysogenes et la notion du provirus, 247 (B)

Bactériocines, v. Colicines

Bactériophage, v. Bacteries lysogenes, Bakteriophage(n)

Bakterienpolysaccharide, leukozyto-taktische Wirkung (Film), 637 (V)

Bakterien- und Zellkulturen, Deck-glasmethode zur längerdauernden kontinuierlichen

Beobachtung, $560(\mathrm{~V})$

Bakteriologie und Serologie, 2. Aufl., 245 (B)

Bakteriophage, v. Antiphagenwirkung, Bacteries lysogenes

Bakteriophagen; Beobachtungen an pleomorphen Proteuskulturen un-ter Einwirkung von

Bakteriophagen, 26

Bakterizidie, v. Gram-stain

ßan </-Agglutinine, Nachweis im Va-ginalsekret von Ban < 7-infizierten Kühen, 539 (V)

Biopsie, v. Organpunktion

Biosynthèse, v. Lactoflavine

Biotin, v. Pflanzlicher Parasit «Blockingtest, umgekehrter», v. Pro-

zonenmechanismus Blood, v. Blutzellen, Katalasebestim- 
mungen, Leukaemia, Leukozyto-

taktische Wirkung, Lewis-System Blutgruppe, v. Lewis-System Blutzellen, lebende, im

Fluoreszenz-

und Phasenkontrastmikroskop,

367 (B) Bovis, v. Bang'-Agglutinine,

Colistämme Bronchialsekret, Cytologie, 365 (B) Brucella abortus, v. Bang-... Brucellose als

Anthropo-Zoonose

(Febris undulans), 126 (B) Budgerigars, v. Psittacosis Butazolidin Geigg, selektiv-insekti-

zide Wirkung, 552 (V)

Cadavre, age, v. Todeszeitbestim-mung

Calonectria graminicola (pflanzlicher Parasit), Beeinflussung von Pathogenität und Wachstum

durch Vitamin Bi und Biotin, 501 (V)

Cancer of the cervix, laboratory diagnosis, 366 (B)

Cancer, v. Bronchialsekret, Ge-schwulstdiagnostik, Kolpomikro-skopie, Leberkrebs

Cancerogene Wirkung; zur Frage einer cancerogenen Wirkung der Azofarbstoffe Evans blue und

Trypanblau auf das retikuloendo-theliale System der Leber, 436

Canís familiaris, v. Mastozytom

Carcinogenese, v. Cancerogene Wirkung

Carcinoma, v. Cancer

Cell wall of bacteries, v. Zellwand

Cell, v. Zell.. .

Cephalgie, v. Migraine

Cerebrum, v. Alzheimer, Pathologi-sche Anatomie

Cervical-Gegend, v. Occipito-Cervical-Gegend

Cervix uteri; laboratory diagnosis of cancer of the cervix, 366 (B)

Cæur, v. Cor

Chemotherapeutica et Antibiotica, Fortschritte (Vol. 2), 366 (B)

Chien, v. Mastozytom

804

Register rerum ad Vol. XIX

Chondriome; modifications physio-logiques et pathologiques du chondriome (Etude au microscope électronique), 217 Chromosomen, v. Letalfaktoren Clostridium tetani, v. Tetanus toxin Cloudy swelling, v. Mitochondries Cobaye, v. Meerschweinchen Colicines; etude de souches coli-cinogènes au microsocope électronique, 582 (V) Colistämme der O-Gruppe 78 mit einem neuen B-Antigen bei der Colisepsis des Kalbes, 543 (V) Collagen diseases, v.

Nephrosclerosis Collumcarcinom, v. Kolpomikrosko-

pie Colony types, v. Corynebakterien Colorants diazoïques, v. Cance-

rogene Wirkung Congrès international des anatomo-pathologistes de langue française, 1956, 248

Congrès, v. Schweizerische Cor, v. Endokarditis, Myokardose Corpse, age, v.

Todeszeitbestimmung Corynebakterien; Typisierung von pathogenen und apathogenen

Corynebakterien auf Grund der Ko-lonienmorphologie, 461 Cow, v. Bang'-Agglutinine

Coxsackie-Viren; erste Nachrichten über in Ligurien gefundene Coxsackie-Viren, 620 (V)

Cryptococcus neoformans, dichroi-

tische Färbung seiner Kapsel, 82 -, Struktur der Kapsel, 305 Culture medium, v. Tetanus toxin

Cultures de tissu, v. Bacillus anthra- 
cís, Zell- und Bakterienkulturen Cyclitols; recherches sur la bio-chimie des cyclitols.

L'utilisation de trois inososes par six micro-organismes, 654 (V) -; v. Anti-inositols

Cytodiagnostik, gynäkologische,

Grundriß und Atlas, 800 (B) Cytodiagnostik, v. Bronchialsekret,

Cancer of the cervix Cytologie des Bronchialsekretes,

365 (B) Cytostaticum, v. Leukaemia

Diabetes insipidus syndrome developed with myelocytic leukaemia, 278

Diagnosis; laboratory diagnosis of cancer of the cervix, 366 (B)

-; mikrobiologische und serologi-sche Diagnostik mit Berücksichti-gung der Pathogenese und Epi-demiologie, 2. Aufl., 244 (B)

-; Organpunktion in der klinischen Diagnostik, 125 (B)

-, v. Bronchialsekret, Cytodiagnostik, Geschwulstdiagnostik, Kolpomikroskopie, Listeriose,

Occipito-Cervical-Gegend

Diazo dyes, v. Cancerogene Wirkung

Dichroitische Färbung der Kapsel des Cryptococcus neoformans, 82

3,5-Dioxo-1,2-diphenyl-4-n-butyl-pyrazolidin Natrium, v. Butazoli-din

Diphtheroid strains, v. Corynebakterien

Dog, v. Mastozytom

Dyes; diazo dyes, v. Cancerogene Wirkung

-Eberthella typhosa, v. Antigène «O»

Elektrizität; Atlas zur Spurenkunde der Elektrizität, 362 (B)

Elektronenmikroskop, v. Colicines, Meningoencephalitis, Mitochondries, Röntgenbestrahlung,

Thyre-oidea

Elektropathologie, v. Elektrizität

Elektrophorese, v. Papierelektro-phoretische ...

Element-trace (Zinc), v. Lactofla-vine

Embryologie, 799 (B)

Encephalitis, v. Meningoencephalitis

Endokarditis, spontane, seröse, des Kaninchens, 318

Endosymbiose, v. Symbionten, Symbiose

Enzymes, v. Ferments

Epidémie hospitalière de staphy-lococcies, recherches bacteriologi-ques, 497 (V)

Epidemiologie der Infektionskrank-heiten, v. Infektionskrankheiten

Register rerum ad Vol. XIX

805

Epidermis; Stimulation der senilen Rattenepidermis durch stabilisierte Amnion-Salbe, 429

-, v. Wundheilung

Erbpathologie, v. Letalfaktoren

Escherichía coli; etude de souches colicinogènes au microscope élec-tronique, 582 (V)

-; isolation of the Streptomycin resistant variants of Escherichia coli B by a modified replica technique, 792

-, v. Colistämme

Evans blue, v. Cancerogene Wirkung

Färbung, v. Dichroitische Färbung,

Gram-stain Farbstoff-Thesaurismose, v. 
Cancerogene Wirkung Febris rheumatica, v. Gelenkveränderungen, Streptokokken Febris undulans, v. Brucellose Feinstruktur, v. Submicroscopical structure Ferments; modifications de Гacti-

vité de quelques systèmes enzyma-

tiques de Torganisme animal pro-

voquées par $\Gamma$ antigène « $\mathrm{O} »$ et $\Gamma$ an-

tigène «Vi», $576(\mathrm{~V})$-, v. Hyaluronidase, Katalase, Kata-

lasebestimmungen, Tetanus toxin Fièvre aphteuse, v. Katalase (Filtres en microscopic et microphotographie, 551 [V]) Fluoreszenz- und Phasenkontrast-

mikroskop; lebende Blutzellen im

Fluoreszenz- und Phasenkontrast-

mikroskop, 367 (B) Foie, v. Liver

Foot-and-mouth disease, v. Katalase France, v. Congrès Freeze-drying, v. Gefriertrocknung

Fungi, dichroitische Färbung der

Kapsel des Cryptococcus neo-

formans, 82 -, v. Calonectña, Cryptococcus neo-

formans, Torulose

Gefriertrocknung, Grundriß, 2. Aufl., 248 (B)

Gelenkveränderungen nach experi-mentellen Streptokokkeninfektio-nen bei Kaninchen, welche

eine

generalisierte Shwartzman-Reak-

tion überlebt haben. II.: Beitrag

zu der experimentellen Forschung

über Streptokokkeninfektionen $\mathrm{u}$.

ihre Folgen, 205 Genetik, v. Alzheimer, Letalfaktoren Genphysiologie der Entwicklung, v.

Letalfaktoren Geschwulstdiagnostik, histologische,

246 (B) Gewerbekrankheiten, 4. Aufl., 245 (B) Gram-stain; factors involved in the

mechanism of Gram-stain, 88 Granulome, sarkoidähnliche, v.

Nephrosclerosis Guinea-pig, v. Meerschweinchen Gynäkologie, v. Kolpomikroskopie

Gynäkologische Cytodiagnostik,

Grundriß und Atlas, 800 (B)

Hämolysegrad, v. Papierelektropho-

retische ... Hamartom, v. Sarcoma idiopathicum Harnblasenparasit, v. Trichoso-

moides Haut, v. Epidermis Hautwunden, Heilung, v. Amnion-

haltige Salbe Heart, v. Endokarditis Hefen, wilde, v. Torulose Hemikranie, v. Migraine Hepar, v.

Cancerogene Wirkung,

Katalase, Katalasebestimmungen,

Kupffer'sche Sternzellen, Leber,

Leberkrebs, Mitochondries,

Regeneration Hepatektomie, v. Regeneration Heredopathie, v. Alzheimer,

Letalfaktoren Herz, v. Endokarditis, Myokardose Histologie, pathologische, v. Patho-

logische Anatomie Histologische Geschwulstdiagnostik,

246 (B) Hôpital, épidémie hospitalière, v.

Staphylocoques Hund; sogenanntes Mastozytom

beim Hund, 249 Hyaline Granula, v. Mitochondries Hyaluronidase, v. Mesenchym-

erkrankungen Hypertension, v. Nephrosclerosis 
806

Register rerum ad Vol. XIX

Hypovitaminose C, chronique, chez le cobaye, lesions articulaires et périarticulaires, 369 Immunbiologie, v. Antikörper

Immunization against plague; (XV. Pathophysiologic action of the toxin of Pasteurella pestis in experimental animals), 51

-; (XVI. Specific neutralization of plague toxin), 71

Immunization, v. Arteriitis

Impfungen, v. Arteriitis

Industrial diseases, v. Gewerbe-krankheiten

Infektionskrankheiten; mikrobiolo-gische und serologische Diagno-stik mit Berücksichtigung der Pathogenese und Epidemiologie, 244 (B)

INH, v. Isonicotinsäurehydrazid-.. .

Inkornplette Antikörper in der Immunbiologie, 247 (B)

Inositol, v. Anti-inositols

Inososes; recherches sur la biochi-mie des cyclitols. L'utilisation de trois inososes par six microorga-nismes, $654(\mathrm{~V})$

Insektizide (selektiv-insektízide) Wirkung von Butazolidin Geigy, 552 (V)

Interstitium und Kapillaren (Symposium 1954), 365 (B)

Intranasale Infektion, v. Strepto-kokken

Isomytilitol, v. Anti-inositols

Isonicotinsäurehydrazid-resistente Tuberkelbakterien, Virulenz beim Meerschweinchen, 710

Italien, v. Coxsackie-Viren, Poliomyelitis

Kälberruhr, v. Colistämme

Kaninchen, v. Endokarditis, Gelenk-veränderungen, Streptokokken

Kapillaren und Interstitium (Symposium 1954), 365 (B)

Kaposi, morbus Kaposi; Sarcoma idiopathicum multiplex haemor-rhagicum, ein Fall, 162

Kapselstruktur von Cryptococcus neoformans, 305

Karger, Dr. Heinz, Dr. med. h. c, 128

Karzinom, v. Cancer (...)

Katalase; Katalaseaktivität der Le-ber beim mit Maul- und Klauen-seuche infizierten

Meerschweinchen (II. Mitt, über Katalase), 98

Katalasebestimmungen beim Meerschweinchen (III. Mitt, über Katalase), 781

Kidney, v. Mitochondries, Renal

Klinische Diagnostik, v. Organ-punktion

Kluyver, Albert Jan, 1888-1956, in memoriam, 488

Koch; les bacilles de Koch incom-plètement évolués dans Гinfection tuberculeuse, 491 (B)

Kollagenkrankheiten, v. Nephro-sclerosis

Kolonienmorphologie, v. Coryne-bakterien

Kolpomikroskopie, Atlas, 801 (B)

Kongenitale Mißbildungen, ihre Ur-sache und Prophylaxe, 492 (B)

Kuh; Nachweis von Berne/-Agglutini-nen im Vaginalsekret ßong--infi-zierter Kühe, 539 (V)

Kupffer'sche Sternzellen; Phagocy-tose lymphatisch leukämischer Zellen in den Kupffer'schen

Sternzellen nach Triaethylenmelamin-behandlung, 748

-, der Rattenleber, 738 
-, v. Cancerogene Wirkung

Laboratory animals, v. Versuchstiere Lactoflavine; recherches sur la bio-synthèse de la lactoflavine. Action du zinc sur la biosynthèse de cette vitamine, 659 (V) Lapin, v. Kaninchen LCL-Körperchen, v. Psittacosis Leber; elektronenmikroskopische Untersuchungen an Rattenlebern nach Röntgenbestrahlung, 150 -; Kupffer'sche Sternzellen der Rattenleber, 738 -, v. Hepar

Leberkrebs, primärer, 798 (B) Leichenserum, v. Papierelektrophoretische ... Le-System, v. Lewis-System Letalfaktoren in ihrer Bedeutung für Erbpathologie und Genphy-siologie der Entwicklung, 368 (B)

Register rerum ad Vol. XIX

807

Leukaemia; diabetes insipidus syndrome developed with myelocytic leukaemia, 278

-; Phagocytose lymphatisch leuk-ämischer Zellen in den Kupffer'-schen Sternzellen nach

Triaethy-lenmelaminbehandlung, 748

Leukozytotaktische Wirkung von Bakterienpolysacchariden (Film), 637 (V)

Levinthal-Coles-Lillie'sche Körper-chen, v. Psittacosis

Lewis-System; über die Sekretions-verhältnisse Neugeborener im Lewis-System (Blut- und Speichel-untersuchungen an 330 Neugebo-renen nebst Bemerkungen über Befunde an 347

Erwachsenen und das Lewis-System im allgemei-nen), 695

Lf (= durch Antitoxin fällbare Sub-stanz), v. Tetanus toxin

«L» forms; formes «L» des Pasteu-rella: Facteurs engendrant leurs transformations, 598 (V)

-; formes «L» des vibrions: Leur pouvoir pathogène, 615 (V)

,- v. Proteuskulturen

Ligurien, v. Coxsackie-Viren, Poliomyelitis

Listeria monocytogenes, $v$. Listeriose

Listeriose, 491 (B)

-, Serodiagnostik, 758

Liver, v. Cancerogene Wirkung, Katalase, Katalasebeslimmungen, Kupffer'sche Sternzellen, Leber, Leberkrebs, Mitochondries, Regeneration

Lobelin und Lobelinalkaloide (Handb. d. exp. Pharmakologie, Bd. 11), 492 (B)

Lungenveränderungen bei Laborato-riumsratten infolge Infektion mit Trichosomoides

crassicauda, 351

Lymphosarkome, v. Thyreoidea

Lyse (Escherichia coli), v. Colicines

Lysogènes; bactéries lysogènes et la notion de provirus, 247 (B)

Mäuse; Adaptationsversuche in Mäu-sen mit Poliomyelitis-Virus Typ 1, $628(\mathrm{~V})$

Maladies industrielles, v. Gewerbe-krankheiten

Man, v. Thyreoidea

Mannitol negative variety, new, of Shigella flexneri 2a, 778

Mastozytom, sogenanntes, beim Hund, 249

Mastzellen, v. Mastozytom

Maul- und Klauenseuche, v. Katalase

Mechanische Einwirkungen, Effekt auf die Zellwand gewisser Bazil-len, 566 (V) 
Meerschweinchen; Katalaseaktivität der Leber beim mit Maul- und Klauenseuche infizierten Meerschweinchen (II. Mitt, über Katalase), 98

-, Katalasebestimmungen (III. Mitt, über Katalase), 781

-, v. Amnionhaltige Salbe, Hypo-vitaminose C, Tuberkelbakterien, Tuberkelbildung

Melopsittacus undulatus, v. Psittacosis

Meningoencephalitis; virus of me-ningoencephalitis in Slovenia, isolation and electron

microscopic morphology, 184

Mesenchymale Zellreaktionen, v. Bacillus anthracis

Mesenchymerkrankungen, neuere Erkenntnisse über ihre Biologie, 798 (B)

Méso-Inositol, v. Anti-inositols

Metabolismus, v. Symbionten

Methode, v. Bakteriologie und Sero-logie, Bronchialsekret, Cancer of the cervix, Cytodiagnostik, Diagnosis, Escherichia coli, Filtres, Fluoreszenz- und Phasenkontrast-mikroskop,

Gefriertrocknung, Ge-schwulstdiagnostik, Kolpomikro-skopie, Mycobactéries, Mycobac-teiïum tuberculosis, Organpunk-tion, Tetanus toxin, Versuchstiere, Zell- und Bakterienkulturen

Microbiology (2nd ed.), 247 (B), 493 (B)

-, perspectives and horizons, 491 (B)

Micrococcus pyogenes var. aureus, in-duzierte Pikromycin-Resistenz, 103

(Microscopie et microphotographie, emploi des filtres, 551 [V])

Register rerur

808

Migraine and vascular allergy,

692 (B) Mikrobiologie, v. Schweizerische Mikrobiologische Diagnostik, v.

Diagnosis Mikroorganismen (Symbiose), v.

Symbionten, Symbiose Mikroskopie, v. Fluoreszenz- und

Phasenkontrastmikroskop, Kolpo-

mikroskopie Mißbildungen, kongenitale, Ursachen

und Prophylaxe, 492 (B) Mitochondries; modifications phy-

siologiques et pathologiques du

chondriome (Etude au microscope électronique) , 217 Morbus Alzheimer, v. Alzheimer Morbus

Kaposi, v. Kaposi-Pick, v. Pick Mus musculus, v. Coxsackie-Virus,

Poliomyelitis Muskel (Herzmuskel), v. Myo-

kardose Mutationen, v. Letalfaktoren Mycobactéries; derniers aspects du

monde des mycobactéries, 493 (B) -, suspensions homogènes, dans $\Gamma$ ex-

périmentation in vitro et in vivo,

504 (V) Mycobacterium tuberculosis; les ba-

cilles de Koch incomplètement

évolués dans Tinfection tubercu-

leuse, 491 (B) -; recherche, isolement et etude du

bacille tuberculeux, 801 (B) -, v. Tuberkelbakterien, Tuberkel-

bildung Myelocytic leukaemia, v. Leukaemia Myokardose, 694 (B)

Nährboden, v. Tetanus toxin

Nase; intranasale Infektion, v. Streptokokken

Necrologia, 488

Nephritis, tuberculous, v. Nephro-sclerosis 
Nephrosclerosis, malignant, and renal tuberculosis (Beport of a case with granulomatous Periarteriitis nodosa, tuberculous nephritis, and hypertension), 13 ‘

$\mathrm{Ne}$ vensystem; Handbuch der spe-ziellen pathologischen Anatomie ad Vol. XIX

und Histologie: 13. Band, 3. Teil

(Zentrales Nervensystem), 363 (B) -, 13. Band, 5. Teil (Peripheres und

vegetatives Nervensystem), 693 (B) -, v. Pathologie, spezielle Neugeborene, v. Lewis-System Neutralization of plague toxin, $\mathrm{v}$.

Plague Newborns, v. Lewis-System Niere, v. Mitochondries, Benal Nouveau-nés, v. Lewis-

System

Oberflächenaktive Substanzen, v.

Tensio-actifs Occipito-Cervical-Gegend, dia-

gnostisch-pathogenetische Studie,

364 (B) Oligoélément (Zinc), v. Lactoflavine Ontogenesis, v. Embryologie,

Letalfaktoren, Mißbildungen Organpunktion in der klinischen

Diagnostik, 125 (B)

Papanicolaou, Untersuchungsmetho-de, v. Bronchialsekret, Cytodia-gnostik

Paper chromatography, v. Tetanus toxin

Papierelektrophoretische Todeszeit-bestimmung aus dem Hämolyse-grad des Leichenserums, Ver-suche, 273

Parasit, v. Pflanzlicher Parasit

Parasite vésical, v. Trichosomoides

Pasteurella, v. «L» forms

Pasteurella pestis, v. Plague

Pathogenese der Infektionskrank-heiten, v. Infektionskrankheiten

-, v. Occipito-Cervical-Gegend

Pathogenität, v. «L» forms

Pathologie; Handbuch der allgemei-nen Pathologie, (Bd. VI/1), 364 (B)

-, spezielle. Pathologie, Pathogenese und Aetiologie wichtiger Krank-heitsbilder (und spezielle Pathologie des Nervensystems), 126 (B)

Pathologische Anatomie und Histologie, spezielle, Handbuch: 13. Band, 3. Teil (Zentrales

Nervensystem), 363 (B)

-, 13. Band, 5. Teil (Peripheres und vegetatives Nervensystem),693 (B)

Register rerum ad Vol. XIX

809

Pathologische Anatomie, spezielle, Lehrbuch, 11./12. Aufl. (Bd. I, Lfg. 3, 4, 5), 127, 128 (B)

Pathologistes, v. Congrès

Peau, v. Epidermis, Wundheilung

Pediculus humanus, v. Butazolidin

Periarteriitis nodosa, granu-lomatous, v. Nephrosclerosis

Peripheres Nervensystem, v. Pathologische Anatomie

Perruche, v. Psittacosis

Personalia, 128

Pest, v. Plague 
Pflanzlicher Parasit (Calonectria graminicola), Beeinflussung von Pathogenität und Wachstum durch Vitamin Bt und Biotin, 501 (V)

Phage, v. Colicines

Phagenwirkung, v. Anliphagenwir-kung

Phagocytose lymphatisch leukämi-scher Zellen in den Kupffer'schen Sternzellen nach

Triaethylenmel-aminbehandlung, 748

Phasenkontrast- und Fluoreszenz-mikroskop; lebende Blutzellen im Fluoreszenz- und

Phasenkontrast-mikroskop,367 (B)

Pick; morbus Pick and morbus Alzheimer, a genetic, clinical, and patho-anatomical study, 244

(B)

Pikromycin-Resistenz, induzierte, bei Micrococcus pyogenes var. aureus, 103

Pilzinfektion, v. Fungi

Plague; immunization against plague (XV. Pathophysiologic action of the toxin of Pasteurella

pestis in experimental animals), 51

-; (XVI. Specific neutralization of plague toxin), 71

Plaies opératoires, guérison, v. Amnionhaltige Salbe

Pleomorphíe, v. Proteuskulturen

Polarisationsmikroskop, v. Dichroi-tische Färbung

Poliomyelitis, 800 (B)

-; Adaptationsversuche in Mäusen mit Poliomyelitis-Virus Typ 1, 628 (V)

-; frequence des anticorps neutrali-sant les trois types de virus polio-myélitique en Ligurie, 633

(V)

-; recherches sur les porteurs de virus poliomyélitique, $624(\mathrm{~V})$

-, v. Coxsackie-Viren

Polysaccharide; leukozytotaktische Wirkung von Bakterienpolysac-chariden (Film), 637 (V)

Portio uteri, v. Kolpomikroskopie

Propylthiouracil, v. Thyreoidea

Proteuskulturen, pleomorphe, Beob-achtungen unter Einwirkung von Bakteriophagen, 26

Provirus, v. Bactéries lysogènes

Prozonenmechanismus; Versuch ei-ner Deutung des Prozonenmecha-nismus mitHilfe eines

«umgekehr-ten Blockingtestes», (II.), 92

Pseudodiphtheriebazillen, v. Coryne-bakterien

Psittacosis; virologischer und histo-logischer Organbefund eines Wel-lensittichs bei Psittacosis, 482

Psychiatrie, v. Alzheimer

Pulmo, v. Bronchialsekret, Lungen-veränderungen

Punktion, v. Organpunktion

Rabbit, v. Kaninchen

Radiation, v. Röntgenbestrahlung

Ratte, v. Epidermis, Leber, Lungen-veränderungen, Kupffer'sche Sternzellen, Thyreoidea

Rattenleber, regenerierende, v. Amnionflüssigkeit

Regeneration; Einfluß einer stabili-sierten Amnionflüssigkeit auf die regenerierende Rattenleber nach partieller Hepatektomie, 288

-; v. Epidermis, Wundheilung

Rein, v. Ren, Renal

Ren, v. Mitochondries, Nephrosclerosis 
Renal tuberculosis and malignant nephrosclerosis (Report of a case with granulomatous Periarteriitis nodosa, tuberculous nephritis, and hypertension), 13

RES, v. Kupffer'sche Sternzellen

Resistenz, v. Pikromycin-Resistenz, Streptomycin

Retikuloendotheliales System der Leber, v. Cancerogene Wirkung

Retothelsarkome, v. Thyreoidea

810

Register rerum ad Vol. XIX

Rheumatismus, Pathogenese, v. Ge-lenkveränderungen, Streptokokken

RHS, v. Kupffer'sche Sternzellen

Riboflavin, v. Lactoflavine

Rind, v. ßani7-Agglutinine, Colistämme

Röntgenbestrahlung; elektronen-mikroskopische Untersuchung an Rattenlebern nach

Röntgenbestrahlung, 150

Saliva, v. Lewis-System

Salmonella typhi, v. Antigène «O»

Sang, v. Rlutzellen

Sarcoid-like granulomas, v. Nephro-sclerosis

Sarcoma idiopathicum multiplex haemorrhagicum (Morbus Kaposi), ein Fall, 162

Sarkome de $\Gamma$ Schilddrüse mit beson-dere $\Gamma$ Berücksichtigu $\pi$ g der Lym-pho- und

Retothelsarkome, 259

Schilddrüse; Sarkome der Schilddrüse mit besonderer Berück-sichtigung der Lympho- und

Retothelsarkome, 259

-, v. Thyreoid(ea)

Schizosaccharomyces Pombe (Lindner) souche liquefaciens (Oster-walder) Dekker, v. Antiinositols

Schütteleffekt, v. Mechanische Ein-wirkungen

Schweizerische Mikrobiologische Ge-sellschaft: 15. Jahresversammlung (1956), 495 ff. (V)

-: Mitgliederliste (1956), $687 \mathrm{ff}$.

Scorbut, v. Hypovitaminose C

Sekretion, v. Lewis-System

Sepsis (Colisepsis der Kälber), v. Colistämme

Serodiagnostik der Listeriose, 758

Serologie und Bakteriologie, 2. Aufl., 245 (B)

-, Fortschritte (Lfg. 15-18), 244 (B)

Serologische Diagnostik, v. Diagnosis

Shigella flexneri $2 \mathrm{a}$, new mannitol negative variety, 778

S/iíüarízman-Reaktion, generalisier-te, v. Gelenkveränderungen, Streptokokken

Sitüch (Wellensittich), v. Psittacosis

Skin, v. Epidermis

- lesions, healing, v. Amnionhaltige Salbe

Skorbut, v. Hypovitaminose C

Slovenia, v. Meningoencephalitis

Société, v. Schweizerische

Speichel, v. Lewis-System 
Spurenelement (Zink), v. Lactoflavine

Staining, v. Dichroitische Färbung, Gram-stain

Staphylocoques; recherches bacté-riologiques à propos d'une épi-démie hospitalière de staphylococcies, $497(\mathrm{~V})$

Stimulation, v. Epidermis, Regeneration

Stomatitis aphthosa (epidemica), v. Katalase

Strahlenwirkung, v. Röntgenbestrahlung

Streptocoques du groupe $A, v$. Antigène $M$ et $C$, Antigène $T$

Streptokokken der Gruppe A, Per-sistenz nach intranasaler Infek-tion, 356

Streptokokkeninfektionen, v. Gelenkveränderungen

Streptomyces felleus, v. Pikromycin-Resistenz

Streptomycin resistant variants of Escherichia coli B, isolation by a modified replica plating

technique, 792

Strommarken, v. Elektrizität

Struma (elektronenmikroskopisch), v. Thyreoidea

Submicroscopical structure, v. Le-ber, Mitochondries, Thyreoidea

Substances tensio-actives, v. Tensio-actifs

Surface active substances, v. Tensio-actifs

Suspensions homogènes, v. Myco-bactéries

Symbionten; experimentelle Analyse der Bedeutung der Symbionten, 665 (V)

(Symbiose; harmonische Einbürge-rung pflanzlicher Mikroorganis-men in den tierischen Körper, $664[\mathrm{~V}])$

Tensio-actifs, anions; action de certains anions tensio-actifs sur le

Register rerum

comportement antigénique des constituants $\mathrm{M}$ et $\mathrm{C}$ des strepto-coques hémolytiques du groupe

A, Ill

-; influence de certains anions ten-sio-actifs sur les reactions d'agglu-tination spécifique étudiées

sur le système T des streptocoques hémolytiques de groupe A, 519 (V)

Teratologie, v. Mißbildungen

Tetanus toxin, large scale production. I.: Toxin production in media prepared with peptic-trypticereptic digested meat or casein, 311

- II.: Chemical research in connection with toxin production, 455

Therapie, v. Antibiotiques, Leukämie

Thesaurismose (Farbstoff-Thesau-rismose), v. Cancerogene W3/8kung

Thiouracil (Propylthiouracil), v. Thyreoidea

Thyroid (Fundamental and clinical text), 493 (B)

Thyreoidea; Morphologie der patho-logisch veränderten menschlichen Schilddrüse im

Elektronenmikro-skop. Vergleich mit der normalen und der durch Propylthiouracil veränderten

Rattenschilddrüse, 129

-; Sarkome der Schilddrüse mit be-sonderer Berücksichtigung d. Lym-pho- und

Retothelsarkome, 259

Tissue culture, v. Bacillus anthracis, Zell- und Bakterienkulturen

Todeszeitbestimmung; Versuche zur papierelektrophoretischen Todeszeitbestimmung aus dem

Hämo-lysegrad des Leichenserums, 273

ToFulopsis neoformans, $v$. Torulose

Torulose, $534(\mathrm{~V})$ 
Toxin, v. Plague, Tetanus toxin

Trace element (Zinc), v. Lacto-flavine

Triaethylenmelaminbehandlung, v. Leukaemia

Trichosomoides crassicauda; Lun-genveränderungen bei Laborato-riumsratten infolge Infektion mit Trichosomoides crassicauda, 351

Trübe Schwellung, v. Mitochondries

Trypanblau, v. Cancerogene Wir-kung

ad Vol. XIX 811

Tuberculosis, v. Renal tuberculosis

-, v. Tuberk ...

Tuberkelbakterien; Virulenz von iso-nicotinsäurehydrazid-resistenten Tuberkelbakterien beim Meer-schweinchen, 710

Tuberkelbildung bei der Meer-schweinchentuberkulose, gleich-zeitiges Einsetzen der Allergie, 1

Tuberkulin, v. Allergie

Tuberkulose; gleichzeitigesEinsetzen von Allergie und Tuberkelbildung bei der

Meerschweinchentuberku-lose, 1

- , v. Mycobacterium tuberculosis

Tuberk ..., v. Tuberc ... .

Tumefaction trouble, v. Mitochondries

Tumoren, v. Bronchialsekret, Cancer of the cervix, Cancerogene Wir-kung, Cytodiagnostik, Geschwulst-diagnostik, Kolpomikroskopie, Le-berkrebs, Mastozytom, Sarcoma idiopathicum, Sarkome der Schilddrüse

Typisierung, v. Colistämme, Coryne-bakterien, Tensio-actifs

Urinary bladder parasite, $\mathrm{v}$. Trichosomoides

Uterus, v. Cervix uteri, Kolpomikroskopie

Vaccination, v. Arteriitis Vache, v. Bang-Agglutinine Vaginalsekret, v. ßang'-Agglutinine Varia, 248 Vascular allergy and migraine,

692 (B) Vasomotorischer Kopfschmerz, v.

Migraine Vegetatives Nervensystem, v. Patho-

logische Anatomie Versuchstiere, Haltung und Zucht,

494 (B) Vibrationen, v. Mechanische Ein-

wirkungen Vibrio, v. «L» forms Virulenz, v. Tuberkelbakterien Virus; Adaptationsversuche in

Mäu-

sen mit Poliomyelitis-Virus Typ 1,

$628(\mathrm{~V})$

812

Register rerum ad Vol. XIX

-; frequence des anticorps neutrali-sant les trois types de virus polio-myélitique en Ligurie, 633

(V)

-; recherches sur les porteurs de virus poliomyélitique, 624 (V)

-; erste Nachrichten über in Ligurien gefundene Coxsackie-Viren, 620 (V)

-, of meningoencephalitis, in Slovenia, isolation and electromicro-scopic morphology, 184

-, Poliomyelitis, 800 (B)

-, v. Antiphagenwirkung, Bactéries lysogènes, Katalase, Psittacosis

Vitamin B $\chi$, v. Pflanzlicher Parasit 
Vitamin B2, v. Lactoflavine

Vitamin $\mathrm{C}$; lesions articulaires et périarticulaires de Thypovitami-nose $\mathrm{C}$ chronique chez le cobaye, 369

Vitamins; Chemistry, Physiology, Pathology (Vol. 3), 362(B)

-; v. Symbionten

Wellensittich, v. Psittacosis Wundheilung, Beschleunigung beim

Meerschweinchen durch amnion-

haltige Salbe, 729 -, v. Regeneration

$\mathrm{X}$-rays, v. Röntgenbestrahlung

Zell- und Bakterienkulturen, Deck-

glasmethode zur längerdauernden

kontinuierlichen Beobachtung,

560 (V) Zellreaktionen, mesenchymale, v.

Bacillus anthiacis Zellwand gewisser Bazillen, Effekt

mechanischer Einwirkungen,

566 (V) Zentralnervensystem, v. Pathologi-

sche Anatomie Zinc, v. Lactoflavine Zyto ..., v. Cyto ...

Register autorum ad Vol. XIX

(B) = Buchbesprechungen - Livre nouveau - Book review

$(\mathrm{V})=$ Vortrag - Communication - Report

Angaben in Klammern ()$=$ Die Arbeit ist nicht erschienen oder wird an einer

anderen Stelle publiziert werden. Indications entre parentheses ()$=$ Le travail n'a pas paru ou

sera publié ailleurs. Notes in parentheses ()$==$ The paper has not been published or will appear elsewhere.

Agnese, G., 624 (V)

Albertini, A. v., 246 (B)

Albertini, A. v., Metaxas, M. N., und

Metaxas-Bühler, M., 1 Antoine, T., und Grünberger, V.,

801 (B) Audrin, J., v. Tison, F.

Baader, E. W., 245 (B)

Banic, S., 792

Barbieri, A. de, et Scevola, M. E.,

576 (V) Barner, F. R., 411 Bartelheimer, H., und Küchmeister,

H. (Editores), 365 (B)

Baumann-Grace, Joyce B., v.

Tomcsik, J. Bednáf, B., v. Raska, K. Beer, K., 534 (V) Bernstein, A., 493 (B) Bloch, H., Gsell, O., und Langer, E.

(Editores), 366 (B) Blondel, B., v. Grasset, E. Bretey, J., v. Nègre, L. Brocher, J. E. W., 364 (B)

Brunner, H. E., 798 (B) Brunner, H. E., v. Rüttner, J. R. (Buchner, P., 664 [V]) Büchner, F., 126

(B) Büchner, F., Letterer, E., und Rou-

let, F. (Editores), 364 (B) 